\title{
Impulse Noise Removal Technique Based on Fuzzy Logic
}

\author{
${ }^{1}$ Mithilesh Atulkar, ${ }^{2}$ A.S. Zadgaonkar and ${ }^{3}$ Sanjay Kumar \\ CV Raman University, Kota, Bilaspur, India \\ 19m.atulkar@gmail.com, 22arunzad28@hotmail.com, ${ }^{3}$ sanraipur@rediffmail.com
}

\begin{abstract}
This paper presents an algorithm to remove random impulse noise in a digital grayscale image based on fuzzy logic approach. Numbers of filters are used to remove noise using fuzzy rules. Filter window used the local statistic to determine local weighted mean. Pixel that are detected noisy are filtered, other pixels are remain unchanged. Experimental results show that this method has better performance than other state of the art filters. The effectiveness of this method is 44.95 in terms of peak-signal-to-noiseratio.
\end{abstract}

Key words: fuzzy logic, image denoising, impulse noise, filter.

\section{Introduction}

In today's information world image act as an information carrier like navigation system traffic observation. Images are captured by use of satellite system video camera, mobile and other acquisition devices. It may likely possible images are corrupted, blurred or degraded due to bad acquisition of images, transmission of images in electromagnetic fields or bad recording[1]. Image consists information and since image is corrupted hence some useful information of in images are removed or image is less informative. Different types of noise are identified; some of them are impulse noise, additive (Gaussian) noise and multiplicative noise. In impulse noise pixel value is replaced by a fixed value either maximum value or minimum value sometimes it may be a random value using random distribution [2]. In additive noise a fixed value is added to each pixel to the image, added value is generated using Gaussian distribution hence it is commonly referred as Gaussian noise. In literature many of them deal with Gaussian noise. In this paper we have to concentrate on impulse noise. During literature many filter found that deal with still image corrupted with noise [3-5]. Several works have been done on fuzzy logic which has better performance compared to non-fuzzy approaches [6-8]. Many filter found that describe video image corrupted by Gaussian (additive) noise [9-12]. Analogously it is necessary to made distinction between filters for color image and gray scale image, filters for gray scale can be used in RGB color image by applying filter on each of the color band separately. Here we present a method for noise removal on a gray scale image.

The paper is structured as follows: in Section 2 describe the methodology and different steps in the actual filtering process are detailed in proposed method Section 3 gives the experimental results. Finally, in Section 4 paper is concluded. 


\section{Proposed Denoising Algorithm}

Consider is a grayscale image to be processed; $w$ is a filtering window of pixel. Filtering window as in figure 2.1 below centered at is surrounded with no of neighbor points of different layer. Points at vertical and horizontal direction of outer layer 1 is stored in and points at diagonals are stored in, similarly points of layer 2 are stored in $\&$ and points of layer 3 are stored in $\&$. In each no of noisy points are find out and stored in an array named.

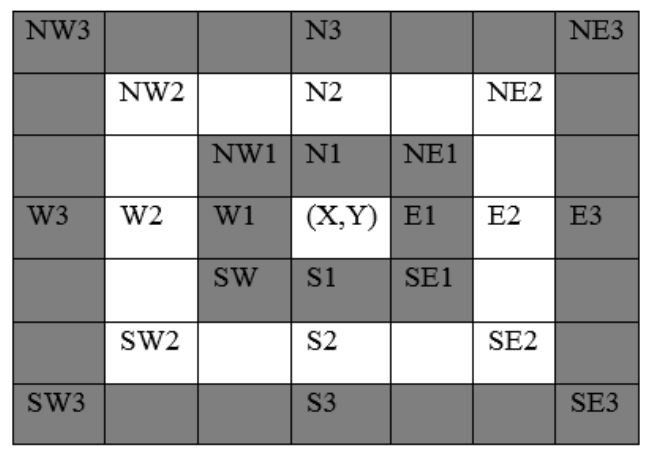

Figure 1: Filter window of size $7 * 7$.

$$
\begin{aligned}
& \operatorname{vec}_{1}=\left[N_{1}, S_{1}, W_{1}, E_{1}\right] \\
& \operatorname{vec}_{2}=\left[N W_{1}, N E_{1}, S W_{1}, S E_{1}\right] \\
& \operatorname{vec}_{3}=\left[N_{2}, S_{2}, W_{2}, E_{2}\right] \\
& v e c_{4}=\left[N W_{2}, N E_{2}, S W_{2}, S E_{2}\right] \\
& \operatorname{vec}_{5}=\left[N_{3}, S_{3}, W_{3}, E_{3}\right] \\
& v e c_{6}=\left[N W_{3}, N E_{3}, S W_{3}, S E_{3}\right] \\
& v e c=\left[v a l_{1}, v a l_{2}, v a l_{3}, v a l_{4}, v a l_{5}, v l_{6}\right]
\end{aligned}
$$

Where, $v a l_{i}$ is no of noisy points in any vector $\left(v e c_{i}\right)$ is calculated by using count () method as described below for $\mathrm{i}=1$ to 6 .

$$
\operatorname{val}_{i}=\operatorname{count}\left(\mathrm{vec}_{i}\right)
$$

$v e c$ is provided as input to the fuzzy inference system to evaluate fuzzy rules. If $F(x, y)$ is corrupted and points of $v e c_{1}$ is noiseless than mean value of $v e c_{1}$ is used to assist the restoration of noisy pixel, but if $v e c_{1}$ is corrupted than $v e c_{2}$ is used to assist new value to noisy point. It is not necessary for any $v e c_{i}$ that all points are noiseless or corrupted. To solve this problem fuzzy logic is used. Fuzzy rules are used to find the $v e c_{i}$, which are responsible to restore the noisy pixel. Filter window is $7 \times 7$ here but for pixel at corner a limited filter of $5 \times 5$ and $3 \times 3$ size is used. 


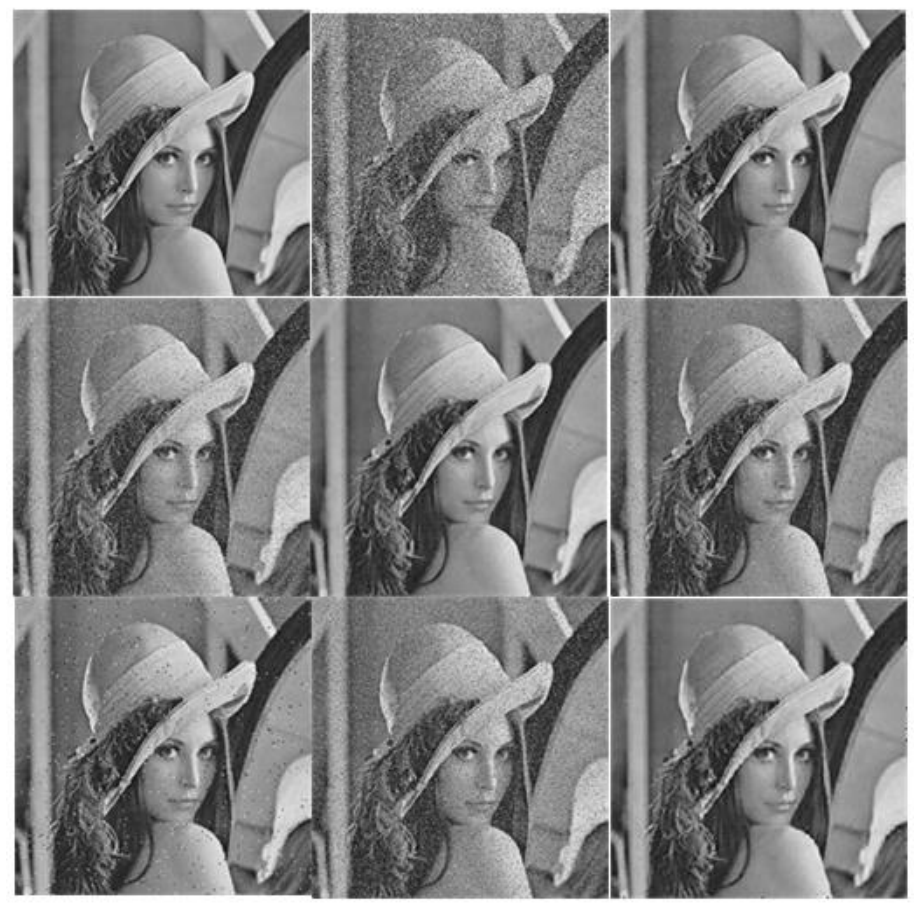

Figure 2: Experimental result with Lena image (a) standard Lena image, (b) noisy image with $30 \%$ impulse noise, Restoration result by corresponding (c) FIRE[16], (d) BDND[17], (e) DWM, (f) LUO, (g) FMEM, (h) SAM, (i) PROPOSED METHOD filters.
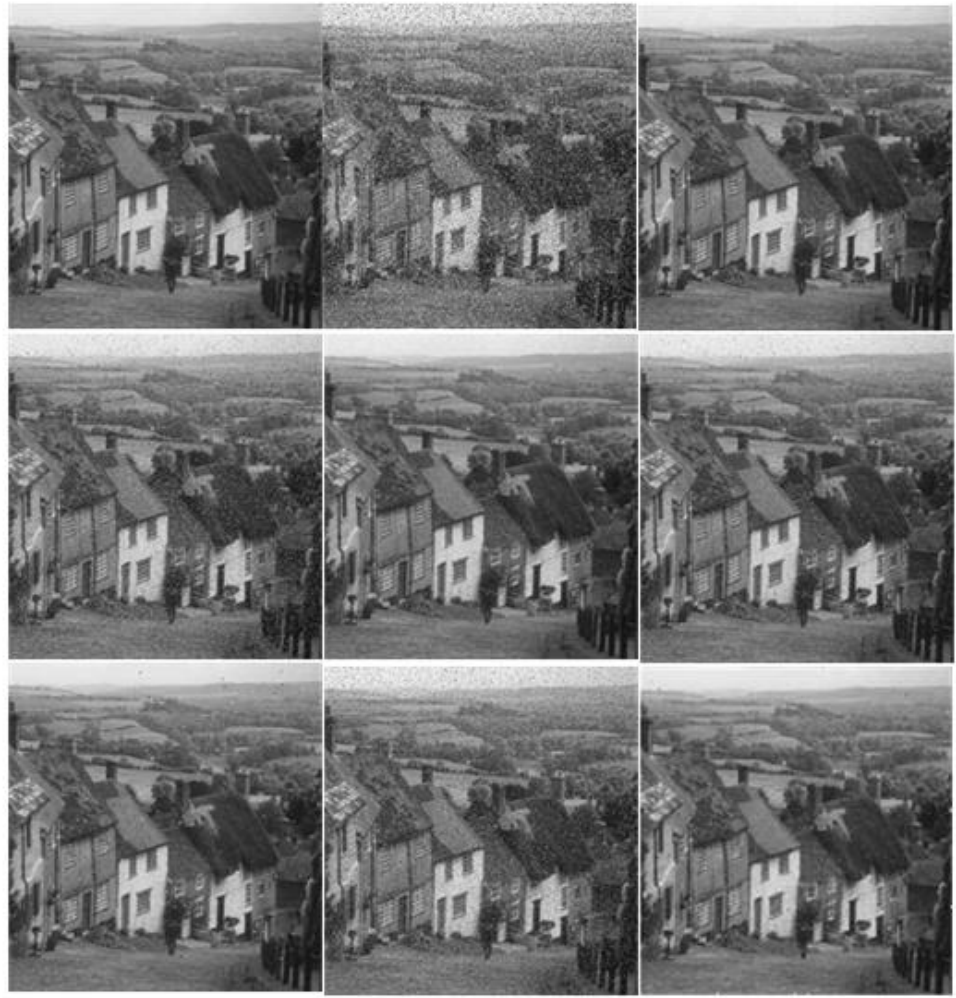

Figure 3: Experimental result with Gold Hill image (a) standard Gold Hill image, (b) noisy image with $30 \%$ impulse noise, Restoration result by corresponding (c) FIRE[16], (d)BDND[17], (e) DWM, (f) LUO, (g) FMEM, (h)SAM, (i) PROPOSED METHOD filters. 
Figure 2 presents visual quality of resultant denoised image of various methods. Figure 2 (a) shows the original lena image. Figure 2 (b) shows 30\% noisy lena image. Figure 2 (i) shows the resultant image of proposed method. Remaining figures are output image of described methods. Figure 3 shows the corresponding original noisy and output image of standard gold hill image.

\section{Result \& Discussion}

Experiment is performed on two standard image of Lena and gold hill image. Value of peak-signal-tonoise-ratio (PSNR) and mean square error (MSE) in images is calculated on the basis of below equation (1) and equation (2).

$$
\begin{aligned}
P S N R & =10 \log _{10}\left(\frac{255^{2}}{M S E}\right)(d b) \\
M S E & =\frac{\sum_{i=0}^{M-1} \sum_{j=0}^{N-1}[y(i, j)-o(i, j)]^{2}}{M . N}
\end{aligned}
$$

PSNR values of denoised image of various methods at different noise density are detailed in table 1 using standard lena image. It is clear from the results that lower the noise density, higher PSNR value. PSNR values are linearly decreasing order with respect to increasing noise level. PSNR value of all methods in table 1 is also calculated in another image gold hill image. In both table 1 and table 2 FIRE and DWM have greater signal to noise ratio corresponding to all other methods.

Table 1: Performance Comparisons of PSNR Value in Lena Image

\begin{tabular}{|l|c|c|c|c|c|}
\hline Method & $\mathbf{1 0 \%}$ & $\mathbf{2 0 \%}$ & $\mathbf{3 0 \%}$ & $\mathbf{4 0 \%}$ & $\mathbf{5 0 \%}$ \\
\hline FIRE [14] & 35.16 & 31.49 & 28.96 & 26.63 & 24.49 \\
\hline BDND [15] & 23.64 & 20.41 & 18.55 & 17.06 & 15.78 \\
\hline DWM [16] & 34.82 & 31.30 & 29.24 & 27.05 & 25.33 \\
\hline LUO [17] & 30.71 & 24.98 & 21.73 & 19.37 & 17.46 \\
\hline FMEM [18] & 34.19 & 28.68 & 23.73 & 20.96 & 17.99 \\
\hline SAM [19] & 22.25 & 19.24 & 17.47 & 16.26 & 15.29 \\
\hline CURRENT METHOD & 44.95 & 42.41 & 41.18 & 39.78 & 38.99 \\
\hline
\end{tabular}

Mean Square Error MSE of resultant denoised image by use of different filtering methods in standard lena image is tabulated in table 3. Unlike PSNR, MSE values are increasing linearly for corresponding noise density. DWM and current methods have better MSE at higher noise level. Table 4 also describes the MSE values for all method in previous table in gold hill image. In gold hill image LUO and current methods have better result. 
Table 2: Performance Comparisons of PSNR Value in Gold Hill Image

\begin{tabular}{|l|c|c|c|c|c|}
\hline Method & $\mathbf{1 0} \%$ & $\mathbf{2 0} \%$ & $\mathbf{3 0} \%$ & $\mathbf{4 0 \%}$ & $\mathbf{5 0 \%}$ \\
\hline FIRE $[14]$ & 34.43 & 31.13 & 28.51 & 26.43 & 24.39 \\
\hline BDND [15] & 23.07 & 19.95 & 18.10 & 16.54 & 15.38 \\
\hline DWM [16] & 34.20 & 30.66 & 28.83 & 26.42 & 24.95 \\
\hline LUO [17] & 30.02 & 24.68 & 21.36 & 19.00 & 17.15 \\
\hline FMEM [18] & 31.90 & 27.52 & 23.32 & 20.57 & 17.71 \\
\hline SAM [19] & 22.02 & 19.08 & 17.27 & 16.04 & 15.05 \\
\hline TLIDE & 33.95 & 29.06 & 23.67 & 20.77 & 16.49 \\
\hline PROPOSED & 44.69 & 42.87 & 40.96 & 39.79 & 38.84 \\
\hline
\end{tabular}

Table 3: Comparison of MSE Value in Lena Image

\begin{tabular}{|l|c|c|c|c|c|}
\hline Method & $\mathbf{1 0 \%}$ & $\mathbf{2 0 \%}$ & $\mathbf{3 0 \%}$ & $\mathbf{4 0 \%}$ & $\mathbf{5 0 \%}$ \\
\hline FIRE [14] & 1.01 & 2.11 & 3.38 & 4.93 & 6.89 \\
\hline BDND [15] & 3.03 & 6.36 & 9.85 & 13.99 & 18.82 \\
\hline DWM [16] & 0.80 & 1.69 & 2.68 & 3.91 & 5.53 \\
\hline LUO [17] & 0.98 & 2.83 & 5.48 & 8.99 & 13.55 \\
\hline FMEM [18] & 1.42 & 2.22 & 3.86 & 6.57 & 10.73 \\
\hline SAM [19] & 3.82 & 7.76 & 11.56 & 13.49 & 19.48 \\
\hline TLIDE & 0.80 & 1.92 & 4.27 & 7.89 & 16.06 \\
\hline PROPOSED & 1.61 & 2.90 & 3.84 & 5.31 & 6.37 \\
\hline
\end{tabular}

Table 4: Comparison of MSE Value in Gold Hill Image

\begin{tabular}{|l|c|c|c|c|c|}
\hline Method & $\mathbf{1 0 \%}$ & $\mathbf{2 0 \%}$ & $\mathbf{3 0 \%}$ & $\mathbf{4 0 \%}$ & $\mathbf{5 0 \%}$ \\
\hline FIRE [14] & 1.10 & 2.28 & 3.61 & 5.22 & 7.27 \\
\hline BDND [15] & 3.38 & 6.98 & 10.80 & 15.36 & 20.27 \\
\hline DWM [16] & 0.98 & 2.09 & 3.37 & 5.01 & 7.06 \\
\hline LUO [17] & 1.26 & 3.26 & 6.12 & 9.88 & 1.46 \\
\hline FMEM [18] & 2.24 & 3.19 & 4.93 & 8.05 & 12.36 \\
\hline SAM [19] & 3.99 & 7.95 & 12.03 & 16.10 & 20.34 \\
\hline TLIDE & 0.98 & 2.27 & 4.78 & 8.43 & 16.60 \\
\hline PROPOSED & 1.71 & 2.61 & 4.05 & 5.31 & 6.60 \\
\hline
\end{tabular}

PSNR, MSE and run time (RT) value of proposed method is described in table 5(a) in standard lena image. Highest PSNR value is 44.95 which is better compare to all other methods as in literature. MSE value should be as possible as smaller, is 1.61 at $10 \%$ noise in lena image. Column 4, RT shows time required in mile seconds to remove impulse noise from the noisy image. Proposed method requires 135 msec at $40 \%$ noise density. Table 5(b) presents corresponding PSNR, MSE and RT values in gold hill image. In order to noise density, PSNR is linearly decreasing and MSE is in increasing order. 
Mithilesh Atulkar ,A.S. Zadgaonkar and Sanjay Kumar; Impulse Noise Removal Technique Based on Fuzzy Logic, Transactions on Machine Learning and Artificial Intelligence, Volume 2 No 6 Dec (2014); pp: 99-105

Table 5: Determination of the PSNR, MSE \& RT Values in (a) Lena Image, (b) Gold Hill Image.

\begin{tabular}{|c|c|c|c|}
\hline Noise level & PSNR & MSE & RT(msec) \\
\hline 10 & 44.95 & 1.616 & 237.7 \\
\hline 20 & 42.41 & 2.90 & 137.57 \\
\hline 30 & 41.18 & 3.84 & 261.51 \\
\hline 40 & 39.78 & 5.31 & 135.70 \\
\hline 50 & 38.99 & 6.37 & 191.15 \\
\hline
\end{tabular}

\begin{tabular}{|c|c|c|c|}
\hline Noise level & PSNR & MSE & RT(msec) \\
\hline 10 & 44.69 & 1.71 & 171.85 \\
\hline 20 & 42.87 & 2.61 & 219.59 \\
\hline 30 & 40.96 & 4.05 & 160.77 \\
\hline 40 & 39.79 & 5.31 & 160.24 \\
\hline 50 & 38.84 & 6.60 & 213.75 \\
\hline
\end{tabular}

\section{Conclusion}

In this paper we present fuzzy logic based noise removal, detail preserving restoration method. This is the ultimate filter for removal of impulse noise. Even at very high noise density image details texture and edges are preserved. The proposed method for image enhancement is sufficient but the future work is to develop a method for image enhancement in RGB color image and video sequenced image.

\section{REFERENCES}

[1]. A. Bovik, Handbook of Image and Video Processing. New York: Academic, 2000.

[2]. Raymond H. Chan, Chung-Wa Ho and Mila Nikolova, "Salt-and-Pepper Noise Removal by Median-Type Noise Detectors and Detail-Preserving Regularization", IEEE Trans. Image Process. October 2005. 14(10): p. 1479-1485. DOI: 10.1109/TIP.2005.852196.

[3]. Tuan-Anh Nguyen, Won-Seon Song, and Min-Cheol Hong, "Spatially Adaptive Denoising Algorithm for a Single Image Corrupted by Gaussian Noise", IEEE Trans. Consumer Electron. August 2010. 56(3): p. 1610-1615.

[4]. R.C. Hardie, C.G. Boncelet, "LUM filters: a class of rank-order-based filters for smoothing and sharpening", IEEE Trans. Signal Process., 1993. 41: p. 1061-1076.

[5]. S.J. Ko, Y.H. Lee, "Center weighted median filters and their applications to image enhancement", IEEE Trans. Circuits Syst., vol. 38, pp. 984-993, 1991.

[6]. S. Schulte, V. De Witte, M. Nachtegael, D. Van der Weken, E.E. Kerre, "Fuzzy random impulse noise reduction method", Fuzzy Sets Syst., 2007. 158: p. 270-283.

[7]. S. Schulte, M. Nachtegael, V. De Witte, D. Van der Weken, E.E. Kerre, “A fuzzy impulse noise detection and reduction method", IEEE Trans. Image Process., 2006. 15(5): p. 1153-1162.

[8]. S. Schulte, S. Morillas, V. Gregori, E.E. Kerre, "A new fuzzy color correlated impulse noise reduction method", IEEE Trans. Image Process., 2007. 16(10): p. 2565-2575.

[9]. S. M. M. Rahman, M. O. Ahmad, and M. N. S. Swamy, "Video denoising based on inter-frame statistical modelling of wavelet coefficients", IEEE Trans. Circuits Syst. Video Technol., 2007. 17(2): p. 187-198. 
[10]. L. Jovanov, A. Pizurica, V. Zlokolica, S. Schulte, P. Schelkens, A. Munteanu, E. E. Kerre, and W. Philips, "Combined wavelet-domain and motion-compensated video denoising based on video codec motion estimation methods", IEEE Trans. Circuits Syst. Video Technol., 2009. 19(3): p. 417-421.

[11]. H. B. Yin, X. Z. Fang, Z. Wei, and X. K. Yang, "An improved motion-compensated 3-D LLMMSE filter with spatio-temporal adaptive filtering support," IEEE Trans. Circuits Syst. Video Technol., 2007 17(12): p. 1714-1727.

[12]. T. Mélange, M. Nachtegael, E. E. Kerre, V. Zlokolica, S. Schulte, V. De Witte, A. Pizurica, and W. Philips, "Video denoising by fuzzy motion and detail adaptive averaging", J. Electron. Imaging, 2008. 17(4).

[13]. Tom Mélange, Mike Nachtegael and Etienne E. Kerre, "Fuzzy Random Impulse Noise Removal From Color Image Sequences", IEEE Trans. Image Process., 2011. 20(4): p. 959-970, DOI: 10.1109/TIP.2010.2077305.

[14]. F. Russo and G. Ramponi, "A fuzzy filter for images corrupted by impulse noise," IEEE Signal Process. Lett., june 1996.3(6): p. 168-170.

[15]. P. E. Ng and K. K. Ma, "A switching median filter with boundary discriminative noise detection for extremely corrupted images," IEEE Trans. Image Process., June 2006. 15(6): p. 1506-1516.

[16]. Y. Dong and S. Xu, "A new directional weighted median filter for removal of random-valued impulse noise," IEEE Signal Process. Lett., Mar 2007. 14(3): p. 193-196.

[17]. W. Luo, "An efficient algorithm for the removal of impulse noise from corrupted images," AEUInt. J. Electron. Commun, 2007. 61: p. 551-555.

[18]. J. Zhang, "An efficient median filter based method for removing random valued impulse noise," Digit. Signal Process, July 2010. 20(4): p. 1010-1018.

[19]. H. Ibrahim, N. S. P. Kong, and T. F. Ng, "Simple adaptive median filter for the removal of impulse noise from highly corrupted images," IEEE Trans. Consumer Electron., Nov. 2008. 54(4): p. 1920-1927. 